Revue internationale P.M.E.

Économie et gestion de la petite et moyenne entreprise

\title{
Informatisation et performance dans la PME au Cameroun
}

\section{Marcellin Henri Ndong Ntah}

Volume 17, numéro 2, 2004

URI : https://id.erudit.org/iderudit/1008458ar

DOI : https://doi.org/10.7202/1008458ar

Aller au sommaire du numéro

\section{Éditeur(s)}

Presses de l’Université du Québec

ISSN

0776-5436 (imprimé)

1918-9699 (numérique)

Découvrir la revue

Citer cette note

Ndong Ntah, M. H. (2004). Informatisation et performance dans la PME au Cameroun. Revue internationale P.M.E., 17(2), 65-92.

https://doi.org/10.7202/1008458ar

\section{Résumé de l'article}

Depuis l'avènement en 1987 du paradoxe de la productivité des investissements en technologies de l'information ou " paradoxe de Solow ", plusieurs études tant macroéconomiques que microéconomiques, adoptant des méthodologies et portant sur des champs divers se sont succédé pour tester la validité empirique de ce paradoxe. Si, sur le plan microéconomique, la plupart des études se sont intéressées aux grandes entreprises, très peu malheureusement ont porté attention aux PME en général et presque pas aux PME africaines en particulier.

C'est dans cette perspective que nous avons décidé, à l'instar de Dans (1999, 2001), Blili et Raymond (1993), Levy et Powell (1988), de contribuer à combler ce vide. Aussi, notre article cherche-t-il à vérifier empiriquement par une enquête sur un échantillon d'une cinquantaine de PME camerounaises si l'informatisation a un impact positif sur leur performance ou, du moins, sur leur productivité. La mesure perceptible de performance utilisée infirme empiriquement le paradoxe de la productivité dans le cadre de notre étude, prouvant ainsi, à la suite de Dans (1999, 2001), Lichtenberg (1995), Brynjolfsson et Hitt (1996) et autres, la caducité de ce paradoxe. Même si nous n'avons pas relevé de corrélation statistique entre informatisation et performance, nous avons conclu notre article en encourageant les PME à s'employer vivement à optimiser l'utilisation de l'ordinateur qui pourrait constituer par là un véritable outil de compétitivité et de performance.
Ce document est protégé par la loi sur le droit d'auteur. L'utilisation des services d’Érudit (y compris la reproduction) est assujettie à sa politique d'utilisation que vous pouvez consulter en ligne.

https://apropos.erudit.org/fr/usagers/politique-dutilisation/ 


\title{
Notes de recherche \\ Informatisation et performance dans la PME au Cameroun
}

\author{
Marcellin Henri NDONG NTAH \\ Programme des Nations Unies pour le développement, Cameroun \\ Université de Douala
}

\section{MOTS CLÉS}

\section{PME - Paradoxe de productivité - Informatisation Performance - Corrélation}

\begin{abstract}
RÉSUMÉ
Depuis l'avènement en 1987 du paradoxe de la productivité des investissements en technologies de l'information ou "paradoxe de Solow", plusieurs études tant macroéconomiques que microéconomiques, adoptant des méthodologies et portant sur des champs divers se sont succédé pour tester la validité empirique de ce paradoxe. Si, sur le plan microéconomique, la plupart des études se sont intéressées aux grandes entreprises, très peu malheureusement ont porté attention aux $P M E$ en général et presque pas aux PME africaines en particulier.

C'est dans cette perspective que nous avons décidé, à l'instar de Dans (1999, 2001), Blili et Raymond (1993), Levy et Powell (1988), de contribuer à combler ce vide. Aussi, notre article cherche-t-il à vérifier empiriquement par une enquête sur un échantillon d'une cinquantaine de PME camerounaises si l'informatisation a un

\section{L'AUTEUR}

Marcellin HenRi NoOng Ntah est docteur d'État en sciences économiques et membre du Laboratoire de recherches GEREA (Groupe d'études et de recherche en économie appliquée) de l'Université de Douala. II est actuellement conseiller en développement au Programme des Nations Unies pour le développement au Cameroun. En plus des thèses de doctorat de $3^{e}$ cycle et de doctorat d'État, il est auteur de plusieurs articles et allocutions présentés à des séminaires, colloques ou conférences et portant sur l'économie d'entreprise, l'économie de développement, l'économie industrielle, la macroéconomie et la pauvreté. Courriels : $<$ marcellin.ndong.ntah@undp.org > et <mhndong@yahoo.fr >.
\end{abstract}


impact positif sur leur performance ou, du moins, sur leur productivité. La mesure perceptible de performance utilisée infirme empiriquement le paradoxe de la productivité dans le cadre de notre étude, prouvant ainsi, à la suite de Dans (1999, 2001), Lichtenberg (1995), Brynjolfsson et Hitt (1996) et autres, la caducité de ce paradoxe. Même si nous n'avons pas relevé de corrélation statistique entre informatisation et performance, nous avons conclu notre article en encourageant les PME à s'employer vivement à optimiser l'utilisation de l'ordinateur qui pourrait constituer par là un véritable outil de compétitivité et de performance.

\section{ABSTRACT}

Since the advent in 1987 of the paradox of investments in information technologies, also known as the "Solow Paradox", several macro- and microeconomic studies drawing on diverse and varied methodologies and fields have followed one another in testing the empirical validity of this paradox. If within the field of microeconomics, the majority of studies have taken an interest in large enterprises, unfortunately very few are dedicated to small and medium enterprises (SME) in general, and almost none to African SME in particular.

It is in this perspective that we have opted to contribute to filling this gap, following the example of Dans (1999, 2001), Blili and Raymond (1993) and Levy and Powell (1988). Our article is concerned with empirically verifying through a survey of a sample of approximately 50 Cameroonian SME if computerisation has a positive impact on their performance or at least on their productivity. The perceptible measure of performance used empirically invalidates the paradox of productivity within the framework of our study, which following Dans (1999 and 2001), Lichtenberg (1995), Brynjolfsson and Hitt (1996) and others, has come to prove the obsolescence of this paradox. Despite the absence of a statistical correlation between computerisation and performance, we conclude our article by encouraging SMEs to energetically apply themselves to optimise the use of computer, which could constitute a real tool of competitiveness and performance.

\section{RESUMEN}

Desde el advenimiento en 1987 de la paradoja de la productividad de las inversiones en tecnologías de la información o "paradoja de Solow », al tomar varios estudios tan macroeconómicos como microeconómicos, y metodologías y campos diversos y variados se sucedieron para someter a un test la validez empírica de esta paradoja. Si sobre el plano microeconómico, el inmensa mayoría de los estudios se interesaron por las grandes empresas, muy poco desgraciadamente se consagraron al PME en general, y casi no al PME africano en particular.

Es en la perspectiva que optamos a ejemplo de Dans (1999 y 2001), Blili y Raymond (1993), Levy y Powell (1988), de contribuir colmando este vacío (hueco). También, se interesa nuestro artículo a verificar empíricamente por encuesta en una muestra de una cincuentena de PME camerunesas si la información tiene un impacto positivo sobre su realización o por lo menos su productividad. La medida perceptible de realización utilizada invalida empíricamente la paradoja de la productividad en el marco de nuestro estudio que viene así para probar en consecuencia de Dans (1999 y 2001), Lichtenberg (1995), Brynjolfsson y Hitt (1996), y 
otros, la caducidad de esta paradoja. A pesar del incumplimiento de una correlación estadística entre informatización y realización, concluímos nuestro artículo animando el PME a emplearse vivamente para optimizar la utilización del ordenador que podría constituir por ahí un instrumento verdadero de competitividad y de realización.

\section{ZUSAMMENFASSUNG}

Seit sich 1987 das Paradox der Produktivität der Investitionen in Informationstechnologien verbreitete, auch das «Paradoxon von Solow genannt, haben mehrere Studien makroökonomisch wie mikroökonomischen Hintergrundes mit verschiedensten methodischen Ansätzen versucht, die empirische Gültigkeit dieses Paradoxons zu testen. Auf mikroökonomischer Ebene hat sich die Mehrzahl der Studien für grosse Unternehmen interessiert, leider sehr wenige fokussierten sich auf KMUs im allgemeinen und praktisch keine Studien widmeten sich den afrikanischen KMUs.

Vor diesem Hintergrund und den Beispielen von Dans (1999 und 2001), Blili und Raymond (1993), Levy und Powell (1988) folgend, wollten wir dazu beitragen, diese Lücke zu füllen. Mit Hilfe einer empirischen Untersuchung bei fünfzig KMU aus Kamerun sollte überprüft werden, ob die Informatisierung eine positive Auswirkung auf ihre Leistung oder zumindest auf ihre Produktivität hat. Im Rahmen unserer Studie konnte mittels der empirisch erfassten und wahrnehmbaren Leistungsmerkmale das Paradox der Produktivität entkräftet werden, so wie in der Folge auch Dans (1999 und 2001), Lichtenberg (1995), Brynjolfsson und Hitt (1996) und andere, die Ungültigkeit dieses Paradoxons bewiesen haben. Obwohl wir in unserer Studie keine Korrelation zwischen Informatisierung und Leistung belegen konnten, schliesst unser Artikel mit einer nachdrücklichen Ermutigung an die KMU, die Nutzung von Computern und anderer Informationstechnologien zu optimieren, da die Informatisierung ein entscheidender Faktor für eine gesteigerte Wettbewerbsfähigkeit und Leistung ist.

\section{Introduction}

En vue d'obtenir un traitement rapide et efficient de l'information et de tirer le meilleur parti de l' «autoroute» de l'information, de nos jours, les entreprises informatisent de plus en plus leur système d'information ${ }^{1}$. Cependant, les expériences en matière d'automatisation du traitement de l'information ou

1. Le système d'information peut se définir comme un système de production, de traitement, de stockage et de diffusion des informations, qui fonctionne avec le système de gestion avec lequel il doit être suffisamment en phase.

Revue internationale P.M.E., vol. 17, nº 2, 2004 
d'informatisation ne sont pas toujours concluantes. En effet, si les avantages de l'automatisation des systèmes d'information sont assez connus ${ }^{2}$, ses coûts sont très souvent mal évalués, voire ignorés à cause essentiellement de la difficulté à les cerner tous.

La littérature nous révèle d'ailleurs qu'après l'euphorie de la décennie 1970 caractérisée par l'acquisition tous azimuts de l'outil informatique et fondée sur la productivité évidente, on observe depuis la fin des années 1980 l'émergence d'une nouvelle rationalité de gestion des technologies de l'information et de la communication (TIC), rationalité combinant les trois volets de la performance d'une entreprise, à savoir l'économie des moyens, l'efficience et l'efficacité 3 (Bouquin, 1991). Il est donc admis depuis la fin des années 1980 que les dépenses en informatique et télécommunications dans les entreprises ne constituent pas en ellesmêmes une garantie de performance. Autrement dit, l'informatisation d'une entreprise aujourd'hui ne conduit pas toujours à l'amélioration de sa performance ou encore le degré d'informatisation n'influence pas toujours significativement le niveau de performance. D'où le paradoxe de la productivité (Brynjolfsson, 1993; Brynjolfsson et Hitt, 1998 ; Dewan et Kraemer, 1998).

La nouvelle rationalité de gestion des TIC s'impose donc actuellement aux entreprises en général et aux PME en particulier. Les PME camerounaises sont d'autant plus concernées que non seulement elles disposent de ressources limitées comme toutes les PME dans le monde, mais elles connaissent en plus, depuis le début des années 1980, une certaine mutation dans leurs habitudes de traitement de l'information.

Aussi, notre étude vise-t-elle essentiellement à s'assurer empiriquement si le paradoxe de la productivité se vérifie ou non dans les PME camerounaises. Nous nous proposons donc de présenter le cadre d'analyse, notamment la méthode d'évaluation de la performance de l'informatisation (1), et de dégager à travers une enquête par questionnaire, l'impact de l'informatisation sur la performance des PME camerounaises (2).

2. Il s'agit notamment de l'amélioration des capacités d'analyse pour une prise de décision, l'accélération de l'accès et de la transmission d'informations, la facilité de mise à jour des informations, l'exactitude et la cohérence des informations, la sauvegarde systématique des données, etc.

3. L'efficacité rapporte le résultat obtenu à un objectif stratégique poursuivi alors que l'efficience rapporte un résultat économique aux moyens. 


\section{Cadre d'analyse}

L'ordinateur a été introduit en gestion dans les années 1960 dans le but d'obtenir des gains de productivité (diminution du coût des services de gestion, raccourcissement des cycles, meilleure gestion des approvisionnements et réduction des stocks, etc.). Toutefois, ces gains n'ont pas toujours été observés aux lendemains de cette automatisation tant au niveau macroéconomique (Franke, 1987 ; Thurow, 1987 ; Roach, 1989 ; David, 1990) qu' au niveau des entreprises (Strassmann, 1990, 1997). D'où le paradoxe exprimé par Solow en 1987 dans une formule désormais célèbre « on voit partout l'âge de l'informatique sauf dans les statistiques de productivité » et baptisée «paradoxe de la productivité ${ }^{4}$ ». Bien que la vérification empirique de ce paradoxe ait suscité depuis lors un vif intérêt chez les chercheurs, on note une difficulté à mesurer l'impact réel des investissements en TIC sur la productivité et la compétitivité des entreprises. Une autre difficulté se pose également lorsqu'il s'agit de l'évaluation globale ou partielle de la performance dans une institution. Aussi importe-t-il de faire le point tant théorique qu'empirique sur ces deux questions afin de disposer de variables et d'une méthodologie pertinentes pour le test empirique du paradoxe de la productivité dans les PME camerounaises.

\subsection{La problématique de l'évaluation de la performance}

Il n'est pas aisé d'évaluer globalement ou même empiriquement la performance d'une organisation. Cette difficulté peut être contournée en appréhendant le mix de la performance tant sur le plan statique, à travers la productivité, que dynamique, à travers la flexibilité (Rowe, 1994).

\subsection{1. Évaluation sous l'angle de la productivité}

Le mix de la performance couvre trois dimensions de la productivité : la productivité en nature, l'efficience et la productivité en valeur.

\section{A. La productivité en valeur}

La productivité en valeur s'obtient en rapportant une production mesurée en valeur monétaire aux ressources consommées. Dans les secteurs à forte densité de maind'œuvre par exemple, il peut s'agir du ratio valeur ajoutée sur salaire. Il peut également s'agir, pour tous les secteurs, de la croissance du chiffre d'affaires par employé.

4. Le «paradoxe de la productivité » peut s'énoncer clairement ainsi : alors que les TIC étaient considérées comme des innovations radicales et perçues comme des outils clés par les entreprises pour accroître leur compétitivité, leur utilisation massive ne se traduisait pas par une amélioration de la productivité telle qu'elle est mesurée dans les statistiques.

Revue internationale P.M.E., vol. 17, nº 2, 2004 
Toutefois, l'évolution de la productivité en valeur est trop tributaire des conditions de marché (prix, fiscalité, comportements opportunistes, etc.) pour qu'elle puisse être uniquement attribuée à l'informatisation. Cela constitue donc une faiblesse majeure de la productivité en valeur en tant qu'instrument pour mesurer l'impact de l'informatisation sur la performance. En s'intéressant à l'expression monétaire de la production, la productivité en valeur s'écarte de l'efficience qui met l'accent sur l'expression physique de la production.

\section{B. L'efficience}

L'efficience vise surtout l'action sur les coûts et notamment sur les coûts unitaires. En effet, lorsqu'on réduit les coûts unitaires, on contribue à l'amélioration de l'efficience. Ainsi, une organisation devient efficiente lorsqu'elle dépense globalement moins pour produire autant, ou lorsqu'elle maintient son niveau de dépense en produisant davantage (Rowe, 1994). On peut l'appréhender en rapportant l'ensemble des ressources consommées à la production physique.

Toutefois, afin de bien différencier l'effet de l'informatisation sur la productivité en valeur et sur l'efficience, il importe de recourir au concept de la productivité en nature.

\section{La productivité en nature}

La productivité en nature s'obtient en rapportant le nombre de quantités produites à l'effectif, et traduit l'action sur le temps d'opérations.

La productivité en nature et la productivité en valeur n'évoluent pas toujours au même rythme; elles peuvent même évoluer en sens inverse. Une étude de Rowe (1994) révèle que la maximisation de la productivité en nature structure les comportements des employés dans le secteur bancaire à cause de l'importance accordée à la codification et à la mesure des temps standards des opérations.

En somme, il apparaît que l'évaluation de la performance soit fortement tributaire de la conception de la productivité. Toutefois, elle peut être affinée en étudiant la dynamique de la productivité à travers le concept de la flexibilité.

\subsection{2. Évaluation sous l'angle de la flexibilité}

Le concept de flexibilité tel qu'il a été développé par Reix (1977) vient compléter celui de la productivité. Il indique, en effet, une aptitude à se maintenir au cours du temps autour des optimums locaux de la productivité et prend sa pertinence dans un environnement perturbé (Rowe, 1994). Il s'ensuit alors une correspondance conceptuelle et même une tension d'efficacité entre les trois dimensions 
de la productivité mentionnées ci-dessus et les trois dimensions de la flexibilité que sont la flexibilité de capacité, la flexibilité de variété et la flexibilité concurrentielle.

\section{A. La flexibilité de capacité}

La flexibilité de capacité désigne l'aptitude à rester efficient lorsque les volumes de production se caractérisent par de fortes variations temporelles comme dans un environnement cyclique. Il en découle une tension d'efficacité entre la recherche de l'efficience maximale et celle de la flexibilité de capacité qui vise en fait à maintenir une efficience minimale dans une large plage de conditions de demande. Aussi, la recherche de l'efficience doit-elle s'accompagner au niveau du pilotage (de l'entreprise) de la recherche de la flexibilité de capacité, c'est-à-dire de la possibilité d'ajuster les volumes requis aux demandes du marché sans pénaliser l'efficience.

Rowe (1994) établit que la flexibilité de capacité dépend dans le contexte français, pour un grand nombre de secteurs économiques, de la qualité et de la diversité de l'offre de télécommunications.

\section{B. La flexibilité de variété}

La capacité de variété vise à entretenir une croissance de la productivité en nature (qui ne serait pas menacée) lorsqu'on élargit la gamme de biens et/ou services produits. Elle pose le problème du dilemme productivité-différenciation. Elle traduit en fait l'aptitude de l'entreprise à accroître la variété de ses biens et / ou services.

\section{La flexibilité concurrentielle}

La flexibilité concurrentielle décrit l'aptitude à maintenir une productivité en valeur dans un environnement mouvant. Elle traduit plus précisément la capacité de l'entreprise à s'adapter à son environnement marchand. Tout comme la productivité en valeur, la flexibilité concurrentielle est partiellement fonction des facteurs difficilement contrôlables par l'entreprise ${ }^{5}$, ce qui limite sa portée en tant qu'instrument de mesure de l'impact de l'informatisation sur la performance de l'entreprise.

Ce point de littérature sur l'évaluation de la performance nous a permis de relever les variables pertinentes susceptibles de refléter la performance d'une organisation.

5. En l'occurrence l'action des concurrents.

Revue internationale P.M.E., vol. 17, nº 2, 2004 


\subsection{La problématique de l'évaluation de l'impact des investissements en TIC dans la performance}

Notre analyse de cette problématique fait essentiellement le point sur les études portant sur l'impact des $\mathrm{TIC}^{6}$; elle guidera notre démarche empirique.

En effet, depuis la fin de la décennie 1980, plusieurs études adoptant des méthodologies variées et portant sur des champs divers ont été menées et peuvent aujourd'hui être évaluées (GMV Conseil, 2000 ; Dans, 1999, 2001 ; Kraemer et Dedrick, 2001).

\subsubsection{Les études sur les données agrégées}

Les études sur les données agrégées sont pionnières dans le domaine et portent surtout sur l'ensemble de l'économie d'un pays. De nombreux économistes, à l'instar notamment d'Oliner et Sichet (2000) et Jorgenson et Stiroh (2000), relèvent à partir des années 1994-1995 un impact positif des investissements en informatique sur la croissance de l'économie. Ils justifient les résultats négatifs des études précédentes, notamment l'existence du paradoxe de Solow, par le fait qu'il faut de grands changements au niveau du stock de capital pour que les impacts de ces changements soient significatifs et puissent se mesurer sur la production. Ce qui ne fut pas le cas jusque-là, car le poids des investissements en TIC était resté faible par rapport aux autres investissements.

Toutefois, partant de l'étude des mécanismes soutenant la «nouvelle économie », plusieurs économistes tels que Gordon (1999) font remarquer que si les TIC sont fondamentales pour les entreprises, il ne faut pas y voir le facteur qui permettrait à lui seul de dynamiser la croissance de tous les secteurs d'activité.

La plupart des études menées sur les secteurs industriels donnent des résultats similaires à ceux obtenus par des études réalisées sur l'économie dans son ensemble: confirmation du paradoxe de productivité dans un premier temps et relativisation de ce paradoxe par la suite.

\subsubsection{Les études sur les données individuelles d'entreprises}

Les études sur les données individuelles d'entreprises tendent de plus en plus à démontrer l'impact positif des TIC sur la productivité des entreprises. Pour Brynjolfsson (1993), le produit marginal brut ${ }^{7}$ est plus important pour le capital

6. Une telle analyse peut également être faite à partir d'une série d'entretiens avec les experts en TIC ou d'un audit des projets TIC dans les entreprises (voir notamment GMV Conseil, 2000).

7. Il s'agit plus précisément de l'augmentation de production engendrée pour l'addition d'un dollar d'input. 
informatique que pour les autres types de capitaux. Barua, Kriebel et Mukhopadhyay (1995) soulignent que les investissements en TIC améliorent quelques indicateurs de performance (la part de marché par exemple), mais pas la productivité. Brynjolfsson et Hitt, en prolongeant leurs travaux, concluent que les investissements en TIC donnent lieu à un produit marginal conséquent (Hitt et Brynjolfsson, 1994 ; Brynjolfsson, 1995 ; Brynjolfsson et Hitt, 1996, 1998). Enfin, selon Greenan et Mairesse (1996), l'impact de l'informatisation sur la productivité est positif, car le «return on investment ${ }^{8}$ » de l'informatique est au moins équivalent à celui des autres formes de capital.

Toutefois, ces études relèvent les limites des analyses économétriques dans ce domaine en ce sens que les aspects immatériels et qualitatifs aussi bien de la production que des facteurs de production sont de plus en plus importants et, par nature, difficiles à chiffrer. Quoi qu'il en soit, nous convenons avec Brynjolfsson et Hitt qu'il est temps de s'interroger davantage sur la meilleure façon d'utiliser l'ordinateur.

Enfin, il faut souligner que la plupart des études relevées ont été effectuées sur les grandes entreprises et très peu sur les PME. Notre étude sur les PME camerounaises vient donc alimenter ce champ de recherches ainsi que la littérature sur l'impact des investissements en TIC sur la performance.

\subsubsection{Bilan des études}

Ce tour d'horizon des multiples études relatives à l'impact des investissements en TIC sur la productivité nous amène aux constats et conclusions suivantes (GMV Conseil, 2000 ; Rowe, 1994 ; Brynjolfsson et Hitt, 1998 ; Kraemer et Dedrick, 2001) :

- le temps qui passe est favorable aux TIC : en effet, alors que les premiers tests concluaient plutôt à un faible impact des nouvelles TIC sur la productivité des entreprises, l'allongement des périodes d'observation affecte positivement le bilan économique de ces technologies ;

- comme dans tout nouveau domaine, il n'existe pas encore d'indicateur de synthèse des nouvelles TIC. Plusieurs chercheurs considèrent que l'investissement physique est insuffisant pour expliquer le phénomène. Aussi importe-t-il d'intégrer les télécommunications, les logiciels et la formation ; cela constitue le meilleur reflet de l'investissement TIC dans les entreprises ;

8. Cette expression se traduit en français par le « retour sur investissement», assimilable au taux de rentabilité de l'investissement. 
- un référentiel commun reste à construire aussi bien pour les indicateurs que pour les méthodologies d'analyse;

- l'évaluation de l'impact des investissements en TIC est non seulement fortement tributaire de la conception de la productivité, mais également du périmètre organisationnel. Elle doit donc intégrer les interdépendances organisationnelles.

En définitive, on peut dire que l'impact de l'informatisation sur la performance n'est pas théoriquement évident ; il est même parfois incertain. Cette incertitude a suscité une curiosité scientifique qui a donné lieu à une abondante littérature sur la vérification empirique du paradoxe de la productivité. Rappelons que la recension de cette littérature constitue une source d'inspiration importante de notre démarche de vérification empirique du paradoxe de la productivité des investissements en TIC dans les PME camerounaises.

\section{Mesure de l'impact du traitement automatique de l'information sur la performance des PME camerounaises}

Cette mesure a été réalisée grâce à une enquête par questionnaire ${ }^{9}$ reposant sur une méthodologie précise (2.1.) et qui nous a permis d'obtenir un certain nombre de résultats (2.2.).

\subsection{Méthodologie de notre cadre empirique du travail}

La PME étant l'unité d'échantillonnage de notre travail empirique, nous avons préalablement essayé de la définir. D'une manière générale, la PME peut être appréhendée tant quantitativement, par son effectif employé, son chiffre d'affaires, son niveau des capitaux propres et même son niveau d'investissement, que qualitativement (Silem, 1994) comme étant des entreprises juridiquement et financièrement indépendantes dans lesquelles le dirigeant assume la responsabilité financière, technique et sociale, sans que ces éléments soient dissociés. Les seuils habituellement retenus pour les critères quantitatifs varient souvent d'un pays à l'autre et parfois même à l'intérieur d'un même pays, comme c'est le cas au Cameroun où coexistent plusieurs définitions de la PME en fonction des utilisateurs ${ }^{10}$. L'absence d'une définition unique et harmonieuse ne rend pas aisé tout exercice

9. Notre enquête a été réalisée au cours des mois d'avril et de mai 2001.

10. Les définitions les plus usuelles sont celles du FOGAPE (Fonds d'aide et de garantie à la PME), de la BEAC (Banque des États de l'Afrique centrale), du Code des investissements et du ministère des Finances. 
d'identification des $\mathrm{PME}^{11}$ au Cameroun. Néanmoins, sur la base des critères retenus $^{12}$ dans les définitions usuelles et de la possibilité d'accéder facilement aux informations relatives à ces critères, nous avons considéré comme PME dans notre travail les unités de production satisfaisant aux conditions suivantes :

- être dirigée par les nationaux;

- être la propriété des nationaux;

- avoir un effectif de 150 personnes au plus ;

- avoir un chiffre d'affaires inférieur à 1000 millions de francs CFA, soit 1524490 euros $^{13}$.

Bien que ne disposant pas d'un cadre d'échantillonnage précis répondant au profil de PME retenu pour notre travail, nous avons pu obtenir des répertoires et annuaires de quelques syndicats professionnels tels que le GICAM (Groupement interpatronal du Cameroun) et le SYNDUSTRICAM (Syndicat des industriels du Cameroun) des informations pertinentes pour la constitution de notre échantillon de travail.

Pour le FOGAPE, par exemple, est réputée PME camerounaise, toute entreprise individuelle ou collective, quelle que soit sa forme juridique dont (Article 8 du décret No 84/510 du 13 juin 1984 portant création et organisation du FOGAPE) : $51 \%$ au moins du capital et les dirigeants sont camerounais; le chiffre d'affaires inférieur ou égal à 1 milliard de CFCA (soit 1524490 euros); les investissements cumulés n'excèdent pas 500 millions de FCFA (soit 762245 euros); les encours de crédits par caisse à court terme ne dépassent pas 200 millions de FCFA (soit 304898 euros).

Pour la BEAC, par PME nationale, il faut entendre toute entreprise dont (Article 5 de l'arrêté MINFI No 0244 du 5 avril 1989) : la majorité des capitaux et les dirigeants sont nationaux; le chiffre d'affaires est inférieur ou égal à 500 millions de FCFA (soit 762245 euros); les fonds propres ne dépassent pas 100 millions de FCFA (soit 152449 euros); les encours de crédit par caisse à court terme ne dépassent pas 100 millions de FCFA (soit 152449 euros).

Les définitions du Code des investissements et du ministère des Finances reposent presque sur les mêmes critères que les précédentes définitions, mais ont des seuils différents. En somme, on peut dire que l'ensemble des définitions courantes de la PME camerounaise tendent à privilégier l'approche quantitative. Mais celles-ci ne sont pas toujours uniformes et varient selon les utilisateurs. Et parfois, chez le même utilisateur, la définition a évolué avec le temps, comme dans le cas du ministère des Finances.

11. Conscients de cette situation, les pouvoirs publics ont commandé en 2002, avec l'appui de la coopération française, une étude visant à proposer une définition cohérente, opérationnelle et unique de la PME au Cameroun.

12. Il s'agit du taux de participation des nationaux, du niveau de fonds propres, d'un niveau des investissements, du chiffre d'affaires, du coût de création d'un emploi et des encours de crédit.

13. En nous basant sur le taux de change suivant : 1 euro $=655,957$ FCFA.

Revue internationale P.M.E., vol. 17, nº 2, 2004 


\subsubsection{Méthode d'échantillonnage et taille de l'échantillon}

Compte tenu de l'absence de cadre d'échantillonnage, nous avons opté pour l'échantillonnage non probabiliste et, plus précisément, pour une procédure de jugement en prenant un certain nombre de précautions au regard de la taille, de la distribution sectorielle et de la distribution spatiale afin de garantir la représentativité de notre échantillon.

Une cinquantaine de questionnaires ont été administrés, mais seulement 42 ont été validés. Avec une telle taille supérieure à 30, la distribution d'échantillonnage de la moyenne peut suivre une distribution normale.

La distribution sectorielle de notre échantillon de questionnaires validés s'étend, comme l'indique le tableau suivant, à toutes les branches d'activité dans lesquelles les PME telles que nous les avons définies sont présentes dans les métropoles de Douala et Yaoundé qui regroupent environ $80 \%$ des PME du Cameroun (Gauthier, 1995). Il semble difficile d'affirmer que les proportions sectorielles utilisées reflètent correctement la réalité nationale actuelle, car les statistiques disponibles dans ce domaine datent encore de l'année $1992^{14}$ alors que le paysage des PME a beaucoup évolué depuis lors du fait des textes de libéralisation de la décennie 1990.

14. En effet, d'après une enquête réalisée en 1992 par le Centre de coopération CamerounCanada sous les auspices de l'ACDI, il existait au Cameroun entre 900 et 950 PME ayant de 15 à 250 employés. Cinquante pour cent d'entre elles se trouvaient à Douala et $20 \%$ à Yaoundé; le reste étant reparti entre les autres grandes villes du pays. Les activités étaient distribuées comme suit: commerce et hôtellerie (33\%), industries manufacturières $(32 \%)$, banques et assurances $(8 \%)$, BTP $(8 \%)$, agriculture $(7 \%)$, services marchands (6\%), transports $(5 \%)$ et industrie extractive (1\%). Quarante-six pour cent des PME étaient constituées sous forme de SA, $30 \%$ sous forme de SARL et $24 \%$ sous forme d'entreprises individuelles. Enfin, il faut dire que toutes ces informations seront actualisées prochainement par les résultats d'une étude exhaustive sur les PME récemment initiée par la Direction de la statistique du ministère des Affaires économiques, de la Programmation et de l'Aménagement du territoire (MINEPAT). 


\section{TABLEAU 1}

Distribution sectorielle de notre échantillon de questionnaires validés

\begin{tabular}{lcc}
\hline Branches d'activité & $\begin{array}{c}\text { Fréquence absolue } \\
(\mathbf{\%})\end{array}$ & $\begin{array}{c}\text { Fréquence relative } \\
(\mathbf{\%})\end{array}$ \\
\hline Industries agroalimentaires et pêche & 3 & 7,1 \\
Industries manufacturières & 7 & 16,7 \\
Commerce-hôtels & 16 & 38,1 \\
Établissements financiers (coopératives & & \\
d'épargne et de crédit, assurance) & 7 & 16,7 \\
Services (transport, informatique, transit, etc.) & 9 & 100 \\
Total & 42 & \\
\hline
\end{tabular}

Source : Notre enquête.

\subsection{2. Élaboration du questionnaire}

Notre questionnaire a été élaboré à partir d'une recherche exploratoire menée auprès d'une demi-douzaine de PME appartenant à notre champ d'étude. Il comporte des questions fermées, qui sont les plus nombreuses, quelques questions ouvertes et des questions semi-fermées ${ }^{15}$. Il comprend trois parties correspondant aux variables conceptuelles de notre analyse, à savoir l'identification, le degré d'informatisation et le niveau de performance. Ces variables sont éclatées en plusieurs variables opérationnelles.

C'est ainsi que l'identification est appréhendée par les variables telles que l'effectif employé, la forme juridique, la branche d'activité, le niveau du chiffre d'affaires, le montant du capital social. De même, le degré d'informatisation est déterminé à l'aide de plusieurs variables opérationnelles dont les plus importantes sont le nombre de micro-ordinateurs, le nombre de serveurs, le nombre de gros ordinateurs, l'étendue de la pratique de l'information dans la PME et les usages de l'informatique.

Suivant notre schéma global d'analyse, le niveau de performance est établi à partir des différentes dimensions de la productivité (statique et dynamique). Ainsi, la productivité en valeur est appréhendée à travers l'évolution de la valeur ajoutée et celle du chiffre d'affaires. L'efficience est mesurée par l'évolution du coût de traitement de l'information et celle des dépenses administratives. La productivité en nature est évaluée par l'évolution des facteurs suivants : vitesse de traitement de l'information, l'accès et la transmission d'informations, les conditions de travail, les blocages humains à la transmission des informations, la diffusion des informations, le volume de la paperasserie et les capacités d'analyse pour la prise

15. Voir annexe I.

Revue internationale P.M.E., vol. 17, $\mathrm{n}^{\mathrm{o}}$ 2, 2004 
de décision. Trois questions dont deux fermées et une ouverte ont été consacrées à la flexibilité de capacité. Deux questions, l'une fermée et l'autre ouverte, portent sur la flexibilité de variété. Nous n'avons pas évoqué la flexibilité concurrentielle dans le questionnaire, car notre schéma global d'analyse nous a révélé qu'avec cet indicateur, tout comme avec la productivité en valeur, le degré d'informatisation n'influence pas significativement le niveau de performance à cause des effets d'autres facteurs, souvent même exogènes à l'entreprise (Rowe, 1994).

L'idée d'utiliser les variables nominales pour mesurer la performance nous est venue essentiellement de notre recherche exploratoire qui nous a permis de constater que les dirigeants des PME au Cameroun étaient réticents à fournir le moindre chiffre concernant leur entreprise.

Sur les 55 questionnaires placés auprès des dirigeants des PME, nous avons pu en récupérer 45 , soit un taux de retour d'environ $82 \%$. Ce taux peut être jugé satisfaisant lorsqu' on sait que, dans notre environnement, les dirigeants des PME sont méfiants à l'égard des questionnaires et, comme la plupart des dirigeants, ne disposent pas assez de temps.

Sur les 45 questionnaires reçus des enquêteurs après administration, nous en avons rejeté 3 en raison de leur incohérence et des réponses incomplètes.

Conscients de ces difficultés, nous avons recruté nos enquêteurs exclusivement parmi les étudiants de $3^{\mathrm{e}}$ cycle ayant déjà effectué des enquêtes par questionnaire. Leur rôle dans notre travail consistait à rencontrer les dirigeants des PME pour leur soumettre notre questionnaire tout en se mettant à leur disposition pour toute précision, information et explication dont ils pourraient avoir besoin. En général, ils sont passés en moyenne deux fois dans une $\mathrm{PME}^{16}$ : la première fois pour remettre le questionnaire au dirigeant de la PME et lui apporter les clarifications nécessaires à son sujet (bien-fondé, articulation, contenu, etc.) et une deuxième fois pour récupérer le questionnaire dûment rempli par le répondant. De plus, après l'administration des questionnaires, les enquêteurs nous ont appuyé dans leur traitement statistique.

Ce traitement statistique du questionnaire a été réalisé grâce au logiciel Sphinx Plus. Cela nous a permis, grâce aux tris à plats, aux tris croisés et aux tests d'association d'aboutir à un certain nombre de résultats.

16. Le nombre de passages dans une PME augmentait en cas de rendez-vous manqué. Il faut également souligner à ce niveau que toute l'opération d'administration des questionnaires était sous notre supervision directe afin de nous assurer de sa fiabilité. 


\subsection{Résultats}

Les principaux résultats de notre travail découlent d'un retraitement de notre questionnaire.

\subsubsection{Degré d'informatisation}

Le principal retraitement de notre questionnaire nous a permis d'établir le degré d'informatisation de toutes les PME de notre échantillon. Pour ce faire, nous avons défini deux groupes de PME : les PME fortement informatisées et les PME faiblement informatisées. Ensuite, à partir de cinq variables opérationnelles, nous avons construit une variable agrégée que nous avons nommée « degré d'informatisation ».

Ont été déclarées PME faiblement informatisées toutes les PME remplissant au moins trois des cinq conditions suivantes :

- avoir moins de cinq ordinateurs ;

- ne pas posséder de serveurs;

- ne pas posséder de gros ordinateurs;

- l'usage de l'ordinateur dans la PME est réservé uniquement aux initiés ;

- le portefeuille d'applications de l'informatique de la PME est limité à un seul usage (en l'occurrence la bureautique).

Ont été déclarées PME fortement informatisées toutes les PME remplissant au moins trois des cinq conditions suivantes:

- avoir au moins cinq ordinateurs ;

- posséder au moins un serveur;

- posséder au moins un gros ordinateur;

- l'ordinateur dans la PME est accessible à tous les employés ;

- le portefeuille d'applications de l'informatique de la PME est constitué de deux usages au moins.

Les caractéristiques de ces deux groupes s'inspirent notamment des travaux sur l'informatisation des entreprises (Pradels, 1981 ; Rigaud, 1982 ; Rivard et Talbot, 1990 ; Toukam, 1999).

Les deux groupes ainsi constitués disposent des effectifs suivants : 22 pour les PME faiblement informatisées et 20 pour les PME fortement informatisées. La distribution de notre échantillon par rapport au degré d'informatisation nous a paru homogène en ce sens que $50 \%$ des PME faiblement informatisées, comme 
l'indique le tableau de contingence suivant, n'ont pas élaboré un plan directeur d'informatisation, alors qu' on ne compte que $25 \%$ de PME fortement informatisées dans cette situation.

TABLEAU 2

Degré d'informatisation et existence d'un plan directeur d'informatisation

\begin{tabular}{lccc}
\hline $\begin{array}{c}\text { Existence d'un plan de } \\
\text { mise en place de } \\
\text { l'information }\end{array}$ & Non & Total \\
$\begin{array}{l}\text { Degré } \\
\text { d'informatisation }\end{array}$ & 11 & 11 & 22 \\
\hline Faiblement informatisée & 15 & 5 & 20 \\
Fortement informatisée & 26 & 16 & 42 \\
\hline Total & & & \\
\hline
\end{tabular}

Source : Notre enquête.

De plus, dans aucune PME faiblement informatisée de notre échantillon, l'ordinateur n'est accessible à tous les employés.

Après la construction de la variable agrégée appelée « degré d'informatisation», nous avons regroupé les modalités jugées très proches pour ce qui est des variables mesurant le niveau de performance. Ainsi, les modalités «très sensiblement » et «sensiblement» ont été regroupées en une seule modalité, «fortement ». De même, les modalités «très faiblement» et «faiblement» ont été fusionnées pour donner naissance à la modalité «faiblement».

Ces retraitements ont facilité notre test de vérification empirique du paradoxe de productivité.

\subsubsection{Mesure perceptible de la performance}

D'une manière générale, la lecture des tableaux de contingence entre le degré d'informatisation et le niveau de performance (voir annexe II) nous révèle que, dans la quasi-totalité des cas, les dirigeants des «PME fortement informatisées » ont, plus que leurs homologues des «PME faiblement informatisées », le sentiment d'une plus grande performance de leurs entreprises.

Nous venons ainsi, grâce à une mesure perceptible de la performance, d'infirmer, à la suite de Lichtenberg (1995), Brynjolfsson et Hitt (1996), Dewan et Kraemer (1998), Lohr (1999) et Dans (1999, 2001), le paradoxe de la productivité des investissements en TIC dans les PME camerounaises. Afin d'apprécier la robustesse de ce résultat, nous allons à présent recourir aux tests de corrélation. 


\subsubsection{Tests de corrélation}

Notre variable agrégée ou conceptuelle « degré d'informatisation » est une variable nominale; de même, toutes nos variables de productivité sont des variables nominales. Les techniques quantitatives nous renseignent que le test statistique approprié pour une mesure d'association entre deux variables au niveau de mesure nominale est le test de khi carré $\left(\chi^{2}\right)$.

Toutefois, le khi carré ne s'applique pas dans tous les cas ${ }^{17}$. Lorsque les conditions d'application du khi carré ne sont pas respectées, on peut recourir à d'autres mesures d'association telles que le coefficient de contingence et le coefficient de corrélation $\phi^{18}$.

\section{A. Le test de khi carré}

Le traitement statistique de notre questionnaire dans le logiciel Sphinx Plus nous a permis, comme l'indique le tableau suivant, d'obtenir les valeurs de khi carré et d'aboutir à des conclusions sur l'état de dépendance entre notre variable explicative, à savoir le degré d'informatisation, et nos variables expliquées auxquelles correspondent les différents indicateurs de performance.

Le tableau 3 révèle dans l'ensemble que le degré d'informatisation n'influence pas significativement le niveau de productivité.

Pour ce qui est des indicateurs de flexibilité qui sont des variables binaires, le khi carré n'est pas approprié. Seule la lecture des tableaux de contingence (annexe II) nous permet de conclure que la dépendance n'est pas significative entre ces variables et le degré d'informatisation, car on observe les mêmes tendances dans les deux catégories (faiblement et fortement informatisée) de PME.

En somme, le degré d'informatisation n'influence significativement ni la productivité ni la flexibilité.

17 En effet, lorsque le nombre de degré de liberté est de 1, le test de khi carré n'est applicable que si les fréquences théoriques sont toutes au moins égales à 5 . Si ce nombre est supérieur à 1 , le test du khi carré n'est pas applicable quand plus de $20 \%$ des fréquences théoriques sont inférieurs à 5 ou lorsqu'une fréquence théorique est inférieure à 1 .

18. Le coefficient de corrélation $\phi$ s'applique uniquement dans le cas d'un tableau de données $2 \times 2$. Dans le cadre de notre travail, c'est donc le coefficient de contingence dont la valeur est $\mathrm{C}=\sqrt{\chi^{2} / n+\chi^{2}}$ ( $n$ étant le nombre total d'observations ayant servi à calculer la valeur du khi carré) qui est applicable. Plus l'écart est grand entre la distribution des fréquences observées et celle des fréquences théoriques, plus le degré de tendance entre les deux variables est élevé et plus la valeur de $C$ est élevée. Signalons que $C$ peut être égal à 0 , mais ne devrait pas atteindre la valeur de 1 . 


\section{TABLEAU 3 \\ Mesure de dépendance entre le degré d'informatisation et l'indicateur de performance}

\begin{tabular}{|c|c|c|c|c|c|}
\hline $\begin{array}{l}\text { Variable } \\
\text { expliquée }\end{array}$ & $\begin{array}{l}\text { Variable } \\
\text { explicative }\end{array}$ & $\chi^{2}$ & $\begin{array}{l}\text { Degré } \\
\text { de liberté }\end{array}$ & $1-\mathbf{P}$ & Conclusion \\
\hline $\begin{array}{l}\text { Évolution de la } \\
\text { valeur ajoutée }\end{array}$ & $\begin{array}{l}\text { Degré } \\
\text { d'informatisation }\end{array}$ & 2,20 & 2 & 66,76 & $\begin{array}{l}\text { La dépendance } \\
\text { n'est pas significative. }\end{array}$ \\
\hline $\begin{array}{l}\text { Évolution du } \\
\text { chiffre d'affaires }\end{array}$ & $\begin{array}{l}\text { Degré } \\
\text { d'informatisation }\end{array}$ & 3,73 & 2 & 84,54 & $\begin{array}{l}\text { La dépendance } \\
\text { n'est pas significative. }\end{array}$ \\
\hline $\begin{array}{l}\text { Évolution } \\
\text { de la vitesse } \\
\text { du traitement } \\
\text { de l'information }\end{array}$ & $\begin{array}{l}\text { Degré } \\
\text { d'informatisation }\end{array}$ & 2,13 & 2 & 85,59 & $\begin{array}{l}\text { La dépendance } \\
\text { n'est pas significative. }\end{array}$ \\
\hline $\begin{array}{l}\text { Évolution de l'accès } \\
\text { et de la transmission } \\
\text { d'informations }\end{array}$ & $\begin{array}{l}\text { Degré } \\
\text { d'informatisation }\end{array}$ & 5,09 & 2 & 92,16 & $\begin{array}{l}\text { La dépendance } \\
\text { n'est pas significative. }\end{array}$ \\
\hline $\begin{array}{l}\text { Évolution des } \\
\text { conditions de travail }\end{array}$ & $\begin{array}{l}\text { Degré } \\
\text { d'informatisation }\end{array}$ & 0,98 & 2 & 38,76 & $\begin{array}{l}\text { La dépendance } \\
\text { n'est pas significative. }\end{array}$ \\
\hline $\begin{array}{l}\text { Évolution des } \\
\text { blocages humains } \\
\text { à la transmission } \\
\text { des informations }\end{array}$ & $\begin{array}{l}\text { Degré } \\
\text { d'informatisation }\end{array}$ & 6,47 & 2 & 96,07 & $\begin{array}{l}\text { La dépendance } \\
\text { est peu significative. }\end{array}$ \\
\hline $\begin{array}{l}\text { Évolution de la } \\
\text { diffusion des } \\
\text { informations }\end{array}$ & $\begin{array}{l}\text { Degré } \\
\text { d'informatisation }\end{array}$ & 6,68 & 2 & 96,46 & $\begin{array}{l}\text { La dépendance } \\
\text { est peu significative. }\end{array}$ \\
\hline $\begin{array}{l}\text { Évolution du volume } \\
\text { de la paperasserie }\end{array}$ & $\begin{array}{l}\text { Degré } \\
\text { d'informatisation }\end{array}$ & 1,03 & 2 & 40,37 & $\begin{array}{l}\text { La dépendance } \\
\text { n'est pas significative. }\end{array}$ \\
\hline $\begin{array}{l}\text { Évolution des } \\
\text { capacités d'analyse } \\
\text { pour la prise des } \\
\text { décisions }\end{array}$ & $\begin{array}{l}\text { Degré } \\
\text { d'informatisation }\end{array}$ & 2,10 & 2 & 65,08 & $\begin{array}{l}\text { La dépendance } \\
\text { n'est pas significative. }\end{array}$ \\
\hline $\begin{array}{l}\text { Évolution du coût } \\
\text { de traitement } \\
\text { de l'information }\end{array}$ & $\begin{array}{l}\text { Degré } \\
\text { d'informatisation }\end{array}$ & 2,58 & 2 & 72,51 & $\begin{array}{l}\text { La dépendance } \\
\text { n'est pas significative. }\end{array}$ \\
\hline $\begin{array}{l}\text { Évolution } \\
\text { des dépenses } \\
\text { administratives }\end{array}$ & $\begin{array}{l}\text { Degré } \\
\text { d'informatisation }\end{array}$ & 4,39 & 2 & 88,87 & $\begin{array}{l}\text { La dépendance } \\
\text { n'est pas significative. }\end{array}$ \\
\hline
\end{tabular}

Source : D'après nos calculs (grâce au logiciel Sphinx Plus).

Toutefois, dans plusieurs cas du tableau 4, les conditions d'application du test du khi carré ne sont pas réunies, d'où le recours au coefficient de contingence. 


\section{B. Mesure d'association par les coefficients de contingence}

\section{TABLEAU 4}

Test par les coefficients de contingence

\begin{tabular}{|c|c|c|c|c|c|}
\hline $\begin{array}{l}\text { Variable } \\
\text { expliquée }\end{array}$ & $\begin{array}{l}\text { Variable } \\
\text { explicative }\end{array}$ & $\chi^{2}$ & $n$ & $\begin{array}{c}\text { Coefficient } \\
\text { de contingence }\end{array}$ & Conclusion \\
\hline $\begin{array}{l}\text { Évolution } \\
\text { de la valeur ajoutée }\end{array}$ & $\begin{array}{l}\text { Degré } \\
\text { d'informatisation }\end{array}$ & 2,20 & 39 & 0,237 & $\begin{array}{l}\text { La dépendance } \\
\text { n'est pas significative. }\end{array}$ \\
\hline $\begin{array}{l}\text { Évolution de l'accès } \\
\text { et de la transmission } \\
\text { d'informations }\end{array}$ & $\begin{array}{l}\text { Degré } \\
\text { d'informatisation }\end{array}$ & 5,09 & 40 & 0,356 & $\begin{array}{l}\text { La dépendance } \\
\text { n'est pas significative. }\end{array}$ \\
\hline $\begin{array}{l}\text { Évolution des } \\
\text { conditions de travail }\end{array}$ & $\begin{array}{l}\text { Degré } \\
\text { d'informatisation }\end{array}$ & 0,98 & 41 & 0,152 & $\begin{array}{l}\text { La dépendance } \\
\text { n'est pas significative. }\end{array}$ \\
\hline $\begin{array}{l}\text { Évolution des } \\
\text { blocages humains } \\
\text { à la transmission } \\
\text { des informations }\end{array}$ & $\begin{array}{l}\text { Degré } \\
\text { d'informatisation }\end{array}$ & 6,47 & 40 & 0,373 & $\begin{array}{l}\text { La dépendance } \\
\text { n'est pas significative. }\end{array}$ \\
\hline $\begin{array}{l}\text { Évolution de la } \\
\text { diffusion des } \\
\text { informations }\end{array}$ & $\begin{array}{l}\text { Degré } \\
\text { d'informatisation }\end{array}$ & 6,68 & 42 & 0,370 & $\begin{array}{l}\text { La dépendance } \\
\text { n'est pas significative. }\end{array}$ \\
\hline $\begin{array}{l}\text { Évolution des } \\
\text { capacités d'analyse } \\
\text { pour la prise } \\
\text { de décision }\end{array}$ & $\begin{array}{l}\text { Degré } \\
\text { d'informatisation }\end{array}$ & 2,10 & 41 & 0,220 & $\begin{array}{l}\text { La dépendance } \\
\text { n'est pas significative. }\end{array}$ \\
\hline $\begin{array}{l}\text { Évolution des } \\
\text { dépenses } \\
\text { administratives }\end{array}$ & $\begin{array}{l}\text { Degré } \\
\text { d'informatisation }\end{array}$ & 7,04 & 39 & 0,391 & $\begin{array}{l}\text { La dépendance } \\
\text { n'est pas significative. }\end{array}$ \\
\hline
\end{tabular}

Source : D'après nos calculs.

Le test par les coefficients de contingence vient ainsi confirmer les conclusions du test du khi carré. C'est dire qu'avec notre échantillon le résultat issu de la mesure perceptible de la performance et qui infirme le paradoxe de la productivité résiste difficilement aux tests de corrélation qui sont essentiellement quantitatifs.

\section{Conclusion}

Le principal apport de notre travail est la non-vérification, à partir d'une mesure perceptible de la performance, du paradoxe de la productivité des investissements en TIC dans un échantillon non probabiliste des PME camerounaises. L'absence de corrélation entre l'informatisation et la performance que révèlent les tests statistiques pourrait lier l'infirmation du paradoxe de la productivité dans le cas de notre étude au phénomène de « rationalisation ». En dépit de cela, le principal apport 
de notre travail constitue un résultat important sur un sujet intéressant tant les praticiens que les enseignants-chercheurs et relevant d'un environnement à explorer davantage. Ce résultat contribue également à relativiser le paradoxe de la productivité dans les PME qui constituent un vecteur déterminant de la croissance économique des pays.

Nos travaux de recherche ultérieurs sur le sujet pourraient, à partir d'une base de données plus large des PME camerounaises ${ }^{19}$, constituer un échantillon plus large et assez représentatif des PME et utiliser une méthodologie plus robuste pour obtenir une corrélation stable entre informatisation et performance.

Mais, d'ores et déjà, à partir des résultats de notre étude et ceux d'études menées depuis la fin des années 1980 sur l'impact des TIC, nous pouvons encourager les PME camerounaises à investir, sur la base d'un plan directeur d'informatisation, dans les TIC, car, comme l'affirment Brynjolfsson et Hitt (1998), « il n'est plus temps de s'interroger sur les impacts des TIC sur la productivité, complexes à mesurer et probablement limités s'ils ne sont pas accompagnés de changements organisationnels, mais sur la meilleure façon d'utiliser l'ordinateur ».

19. Une telle base de données pourrait provenir des résultats d'une étude en cours sur les PME que mène la Direction de la statistique et de la comptabilité nationale du MINEPAT (Ministère des Affaires économiques, de la Programmation et de l'Aménagement du territoire). 


\section{Annexes}

\section{Annexe I}

\section{Notre questionnaire}

Le présent questionnaire s'inscrit dans le cadre d'un programme de recherche dont l'objectif principal est d'évaluer l'impact de l'informatisation sur la performance des PME, l'informatisation pouvant être définie comme l'automatisation du traitement de l'information. Tout en vous garantissant la confidentialité de vos réponses, nous vous remercions à l'avance pour votre collaboration.

\section{I - INFORMATIONS GÉNÉRALES}

1 Quelle est la date de création de votre PME ?

2 Dans quelle branche d'activité exercez-vous ?

3 Quelle est la forme juridique de votre entreprise ?
$1 \circ$ SARL
$2 \circ$ Société en nom collectif
$3 \circ$ Société anonyme
$4 \circ$ Autre (précisez)

4 Quel est le syndicat professionnel de votre entreprise ?
$1 \circ$ SYNDUSTRICAM
$2 \circ$ GICAM
$3 \circ$ CCIM
$4 \circ$ Autre (précisez)

5 Combien de personnes employez-vous?

6 Dans quel intervalle se situe votre chiffre d'affaires annuel (en FCFA) ?
1 Inférieur à 25 millions
$2 \circ \quad$ Entre 25 et 75 millions
3 Entre 75 et 100 millions
4 - Entre 100 et 300 millions
$5 \circ$ Entre 300 et 500 millions
6 - Plus de 500 millions

7 Quel est votre capital social (en FCFA)?

\section{II - DEGRÉ D'INFORMATISATION}

8 En quelle année avez-vous commencé à informatiser votre entreprise ?

9 Quel est le nombre de micro-ordinateurs de votre parc informatique?

10 Quel est le nombre de serveurs de votre parc informatique?

11 Quel est le nombre de gros ordinateurs de votre parc informatique?

12 Quelle est la proportion (en \%) du matériel d'occasion dans votre parc informatique?

13 L'informatisation de votre entreprise a t-elle été précédée de l'élaboration d'un plan de mise en place?
$1 \circ$ Oui
$2 \circ$ Non 
14 Si oui, qui a élaboré ce plan ?

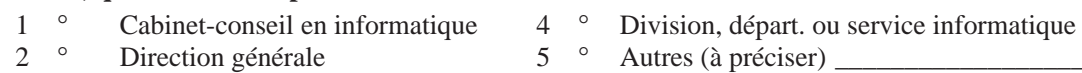

15 Quelle situation reflète le plus la pratique de l'informatique dans votre entreprise ?

1 ○ Accessible à tous les employés $\quad 3{ }^{\circ}$ Autres (à préciser)

2 - Réservée aux initiés uniquement

16 Quelle est la fréquence de participation de vos employés à des séminaires de recyclage en informatique?

$\begin{array}{llllll}1 & \circ & \text { Très souvent } & 4 & \circ & \text { Très rarement } \\ 2 & \circ & \text { Souvent } & 5 & \circ & \text { Jamais } \\ 3 & \circ & \text { Rarement } & & & \end{array}$

17 Quels sont actuellement les usages de l'informatique dans votre entreprise ?

$1 \quad \square \quad$ Bureautique (traitement de texte, tableur, PAO, etc.)

$2 \square$ Applications transactionnelles (facturation, paie, finance, stock, etc.)

$3 \square \quad$ Gestion de bases de données

$4 \quad \square \quad$ Gestion des systèmes télématiques

$5 \square \quad$ Aide à la décision (modélisation, analyse de sensibilité, etc.)

$6 \square$ Générateurs d'application et logiciels de développement

$7 \square \quad$ Jeux et didacticiels

$8 \square \quad$ Autres (à préciser)

\section{III - PERFORMANCE}

18 Depuis l'informatisation de votre entreprise, votre effectif a globalement
1 ○ Diminué
2 - Augmenté

19 S'il a globalement augmenté, on peut qualifier cette augmentation de
1 - Très sensible
$2 \circ \quad$ Sensible
$4 \circ$ Faible
3 Moyenne
$5 \circ$ Très faible

20 Depuis l'informatisation de votre entreprise, on peut dire que votre valeur ajoutée a globalement augmenté
1 ○ Très sensiblement
$2 \circ$ Sensiblement
$4 \circ$ Faiblement
$3 \circ$ Moyennement
$5 \circ$ Très faiblement

21 Depuis l'informatisation de votre entreprise, on peut dire que votre chiffre d'affaires a globalement augmenté
1 ○ Très sensiblement
$2 \circ$ Sensiblement
$4 \circ$ Faiblement
3 - Moyennement
$5 \circ$ Très faiblement

22 Comment est assurée la maintenance de vos équipements informatiques ?
$1 \circ$ Dépannage
$3 \circ$ Autres (à préciser)
$2 \circ$ Contrat

23 Quelle est la fréquence des pannes de vos équipements informatiques?
$1 \circ$ Très souvent
$2 \circ$ Souvent
$4 \circ$ Très rarement
$3 \circ$ Rarement
$5 \circ$ Jamais

24 La maintenance de vos équipements informatiques vous coûte
$1 \circ$ Très cher
$2 \circ$ Cher
$3 \circ$ Presque rien 
25 Depuis l'informatisation de votre entreprise, on peut dire que la vitesse de traitement de l'information dans votre entreprise a d'une façon générale augmenté
$1 \circ$ Très sensiblement
$2 \circ \quad$ Sensiblement
$3 \circ \quad$ Moyennement
$4 \circ$ Faiblement
$5 \circ$ Très faiblement

26 Depuis l'informatisation de votre entreprise, on peut dire que l'accès et la transmission d'informations se sont d'une façon générale améliorés
1 - Très sensiblement
$2 \circ \quad$ Sensiblement
$4 \circ$ Faiblement
3 - Moyennement
$5 \circ$ Très faiblement

27 Depuis l'informatisation de votre entreprise, on peut dire que les conditions de travail se sont d'une façon générale améliorées
1 - Très sensiblement
$2 \circ$ Sensiblement
$4 \circ$ Faiblement
$3 \circ$ Moyennement
$5 \circ$ Très faiblement

28 Depuis l'informatisation de votre entreprise, on peut dire que les blocages humains à la transmission des informations ont d'une façon générale diminué
1 - Très sensiblement
4 Faiblement
$2 \circ \quad$ Sensiblement
$5 \circ$ Très faiblement
$3 \circ$ Moyennement

29 Depuis l'informatisation de votre entreprise, on peut dire que la diffusion des informations s'est d'une façon générale améliorée
1 - Très sensiblement
$2 \circ$ Sensiblement
$4 \circ$ Faiblement
$3 \circ \quad$ Moyennement
$5 \circ$ Très faiblement

30 Depuis l'informatisation de votre entreprise, on peut dire que le volume de la paperasserie a d'une façon générale diminué
1 - Très sensiblement
$4 \circ$ Faiblement
$2 \circ \quad$ Sensiblement
$5 \circ$ Très faiblement
$3 \circ$ Moyennement

31 Depuis l'informatisation de votre entreprise, on peut dire que les capacités d'analyse pour la prise de décision se sont d'une façon générale améliorées
1 - Très sensiblement
$4 \circ$ Faiblement
$2 \circ \quad$ Sensiblement
$3 \circ$ Moyennement
$5 \circ$ Très faiblement

32 Depuis l'informatisation de votre entreprise, on peut dire que le coût de traitement de l'information a d'une façon générale diminué
1 ○ Très sensiblement
$4 \circ$ Faiblement
$2 \circ$ Sensiblement
3 - Moyennement
$5 \circ$ Très faiblement

33 Depuis l'informatisation de votre entreprise, on peut dire que les dépenses administratives ont d'une façon générale diminué
1 - Très sensiblement
$4 \circ$ Faiblement
$2 \circ$ Sensiblement
$3 \circ$ Moyennement
5 - Très faiblement

34 Depuis l'informatisation de votre entreprise, on peut dire que les conditions de travail se sont d'une façon générale améliorées
10
Très sensiblement
$\begin{array}{lll}2 & \circ & \text { Sensiblement } \\ 3 & \circ & \text { Moyennement }\end{array}$
$4 \circ$ Faiblement
5 - Très faiblement

Revue internationale P.M.E., vol. 17, n 2, 2004 
35 S'il vous arrivait aujourd'hui de satisfaire une demande plus importante sur le marché, donc de produire plus, pensez-vous que l'évolution de votre coût de traitement de l'information serait la même?
$1 \circ$ Oui
$2 \circ$ Non

36 Si oui, pourquoi?

37 S'il vous arrivait aujourd'hui de satisfaire une demande plus importante sur le marché, donc de produire plus, pensez-vous que l'évolution de vos dépenses administratives serait la même?
$1 \circ$ Oui
$2 \circ$ Non

38 Si oui, pourquoi?

39 S'il vous arrivait aujourd'hui de répondre à une demande nouvelle sur le marché, donc de produire un autre type de biens et / ou services, pensez-vous que l'évolution de vos temps d'opération (durée du traitement de l'information, temps d'accès à l'information, durée de transmission et de diffusion de l'information) serait la même ?
$1 \circ$ Oui
$2 \circ$ Non

40 Si oui, pourquoi ?

\section{Annexe II}

\section{Quelques tableaux de contingence entre le degré d'informatisation et le niveau de performance}

(N.B. : Les valeurs de nos tableaux sont les pourcentages en ligne établis sur 42 observations).

\begin{tabular}{lllll}
\hline & $\begin{array}{c}\text { Évolution accès et trans- } \\
\text { mission de l'infor- } \\
\text { mation }\end{array}$ & Sensiblement & & \\
$\begin{array}{l}\text { Degré } \\
\text { d'informatisation }\end{array}$ & $45,5 \%(10)$ & $27,3 \%(6)$ & $18,2 \%(4)$ & $\mathbf{1 0 0 , 0} \%(\mathbf{2 0})$ \\
\hline $\begin{array}{l}\text { Faiblement informatisée } \\
\text { Fortement informatisée }\end{array}$ & $75,0 \%(15)$ & $25,0 \%(5)$ & $0,0 \%(0)$ & $\mathbf{1 0 0 , 0} \%(\mathbf{2 0})$ \\
\hline TOTAL & $\mathbf{5 9 , 5 \% ( 2 5 )}$ & $\mathbf{2 6 , 2} \%(\mathbf{1 1})$ & $\mathbf{9 , 5} \%(\mathbf{4})$ & $\mathbf{1 0 0 , 0} \%(\mathbf{4 0})$ \\
\hline
\end{tabular}

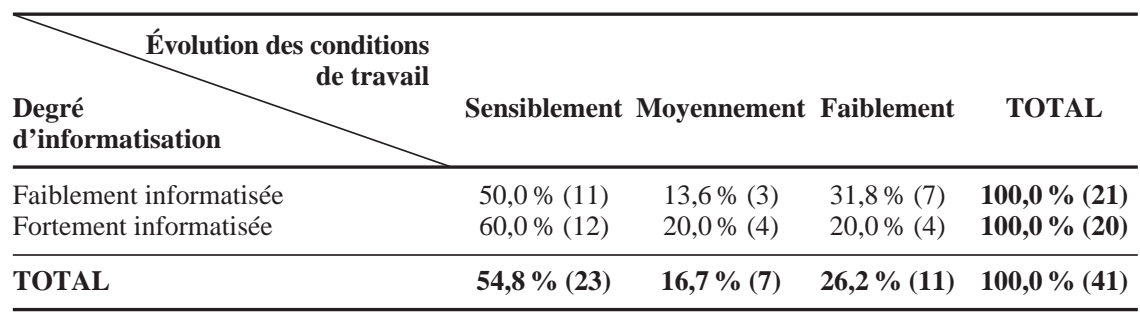




\begin{tabular}{|c|c|c|c|c|}
\hline $\begin{array}{l}\text { Degré } \\
\text { d'informatisation }\end{array}$ & Sensiblement & Moyennement & Faiblement & TOTAL \\
\hline Faiblement informatisée & $27,3 \%(6)$ & $27,3 \%(6)$ & $36,4 \%(8)$ & $100,0 \%(20)$ \\
\hline Fortement informatisée & $70,0 \%(14)$ & $15,8 \%(3)$ & $15,0 \%(3)$ & $100,0 \%(20)$ \\
\hline TOTAL $\quad 47,6 \%(20)$ & $21,4 \%(9)$ & $26,2 \%(11)$ & $100,0 \%(40)$ & \\
\hline $\begin{array}{r}\text { Évolution de la diffusion } \\
\text { des informations }\end{array}$ & & & & \\
\hline $\begin{array}{l}\text { Degré } \\
\text { d'informatisation }\end{array}$ & Sensiblement & Moyennement & Faiblement & TOTAL \\
\hline Faiblement informatisée & $40,9 \%(9)$ & $40,9 \%(9)$ & $18,2 \%(4)$ & $100,0 \%(22)$ \\
\hline Fortement informatisée & $80,0 \%(16)$ & $15,0 \%(3)$ & $5,0 \%(1)$ & $100,0 \%(20)$ \\
\hline TOTAL & $59,5 \%(25)$ & $28,6 \%(12)$ & $11,9 \%(5)$ & $100,0 \%(42)$ \\
\hline $\begin{array}{r}\begin{array}{r}\text { Évolution des capacités } \\
\text { d'analyse pour la prise } \\
\text { des décisions }\end{array} \\
\text { des de }\end{array}$ & & & & \\
\hline $\begin{array}{l}\text { Degré } \\
\text { d'informatisation }\end{array}$ & Sensiblement & Moyennement & Faiblement & TOTAL \\
\hline Faiblement informatisée & $40,9 \% 59^{\circ}$ & $27,3 \%(6)$ & $27,3 \%(6)$ & $100,0 \%(21)$ \\
\hline Fortement informatisée & $65,0 \%(13)$ & $15,0 \%(3)$ & $20,0 \%(4)$ & $100,0 \%(20)$ \\
\hline TOTAL & $52,4 \%(22)$ & $21,4 \%(9)$ & $23,8 \%(10)$ & $100,0 \%(41)$ \\
\hline $\begin{array}{l}\text { Degré } \\
\text { d'informatisation }\end{array}$ & Sensiblement & Moyennement & Faiblement & TOTAL \\
\hline Faiblement informatisée & $18,2 \%(4)$ & $31,8 \%(7)$ & $36,4 \%(8)$ & $100,0 \%(19)$ \\
\hline Fortement informatisée & $20,0 \%(4)$ & $10,0 \%(2)$ & $70,0 \%(14)$ & $100,0 \%(20)$ \\
\hline TOTAL & $19,0 \%(8)$ & $21,4 \%(9)$ & $52,4 \%(22)$ & $100,0 \%(39)$ \\
\hline
\end{tabular}




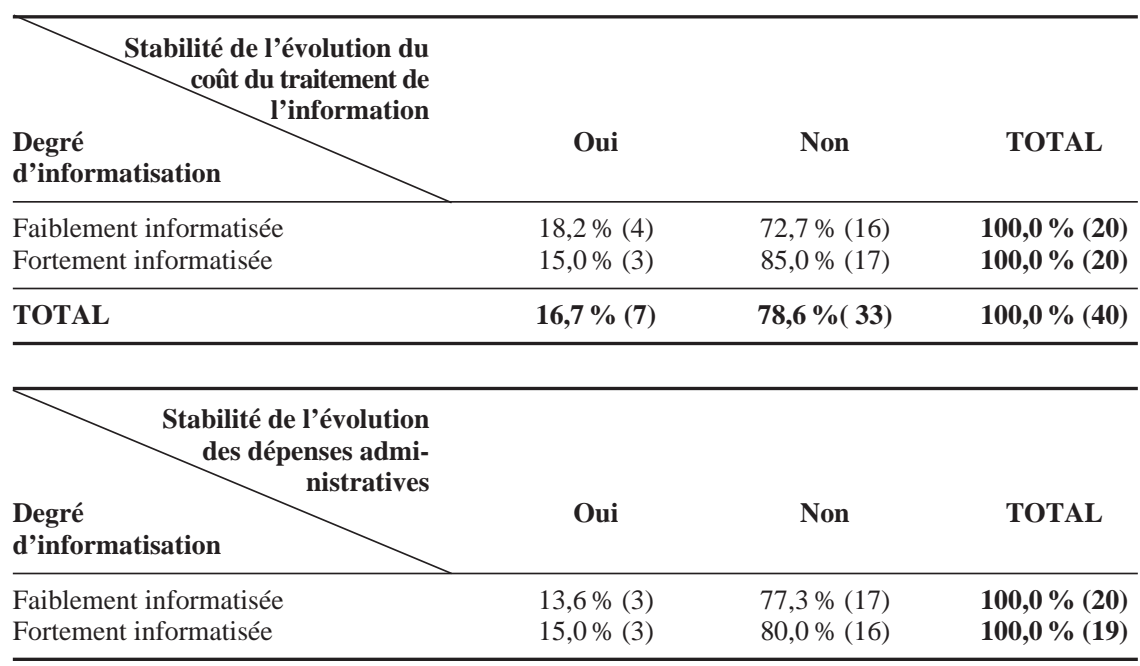

\section{Bibliographie}

Barua, A., C.H. Kriebel et T. MuKhopadhyay (1995), «Information technologies and business value : an analytic and empirical investigations », Information Systems Research, vol. 6, $\mathrm{n}^{\circ}$ 1, p. 3-23.

BLILI, S. et L. RAYMOND (1993), «Information technology : threats and opportunities for small and medium-sized enterprises », International Journal of Information Management, vol. 13, p. 439-448.

BouQuin, H. (1991), Le contrôle de gestion, Paris, Presses universitaires de France.

BRYNJOLFSSON, E. (1993), "The productivity paradox of information technology", Communications of the ACM, vol. 36, $\mathrm{n}^{\circ} 12$, p. 67-77.

BRYNJOLFSSON, E. (1995), «The contribution of information technology to consumer welfare », Working paper MIT Sloan School of Management.

BRYNJOLFSSON, E. et L. HitT (1996), « Paradox lost? Firm level evidence on the returns to information systems spending », Management Science, vol. 42, no 4, p. 541-558.

BRyNJOLFSSON, E. et L. HITT (1998), «Beyond the productivity paradox », Communications of the ACM, vol. 41, no 8, p. 49-55.

DANS, E. (1999), «IT investment in small and medium enterprises: paradoxically productive? », IS Working Papers, The Anderson School of Management at UCLA.

DANS, E. (2001), «IT responsiveness in small and medium enterprises : it pays to be on top IT », Working paper, Instituto de Empressa, Madrid, Espagne.

DAVID, P. (1990), «The dynamo and the computer : an historical perspective on modern productivity paradox », The American Economic Review, vol. 80, n 2, p. 355-361. 
DEWAN, S. et K. KRAEMER (1998), «International dimensions of the productivity paradox », Communications of the ACM, vol. 41, no 8, p. 56-62.

FRANKE, R. H. (1987), «Technical revolution and productivity decline : computer introduction in the financial industry », Technical Forecast Society Change, $\mathrm{n}^{\circ} 31$.

GAUTHIER, B. (1995), «La dynamique des entreprises manufacturières au Cameroun », dans Cahiers CETAI / HEC, Montréal.

GMV CONSEIL - CABINET CONSEIL ET ÉTUdES MARKETING DE PARIS (2000), «L'impact des NTIC sur la compétitivité des entreprises industrielles », étude présentée à la CPCI (Commission permanente de concertation pour l'industrie), Ministère de l'Économie, des Finances et de l'Industrie, France.

GORDON, R.J. (1999), «Has the new economy rendered the productivity slowdown obsolete? », Cahier de recherche, Northwestern University \& NBER.

GREENAN, N. et J. MAIRESSE (1996), «Computers and productivity in France : some evidence », NBER Working Paper, $\mathrm{n}^{\circ} 5836$.

HiTT, L. et E. BRYNJOLFSSON (1994), «The three faces of IT value : theory and evidence », dans J.I. Degross, S.L. Huff et M.C. Munro (dir.), Actes de la $15^{e}$ International Conference on Information Systems, 14-17 décembre, Vancouver, Canada, p. 263-289.

JorGEnSON, D. et K.J. STIROH (2000), «Raising the speed limit : U.S. economic growth in the information age », $1^{\mathrm{er}}$ mai ; disponible sur : 〈kwhelen@ frb.org>.

KRAEMER, K. et J. DEDRICK (2001), « The productivity paradox : Is it resolved ? Is there a new one ? What does it all mean for managers?», Document de travail, Centre for Research on Information Technology and Organisations, University of California, Irvine, États-Unis.

LEVY, M. et P. POWELL (1998), «SME flexibility and the role of information systems », Small Business Economics, vol. 11, p. 183-196.

LiCHTENBERG, F. (1995), «The output contributions of computer equipment and personnel : a firm level analysis», Economic Innovations and New Technology, vol. 3, p. 201-217.

LOHR, S. (1999), «Computer age gains respect of economists », The New York Times, 14 avril.

OLINER, S. et D. SICHEL (2000), The Resurgence of Growth in the Late 1990s : Is Information Technology the Story?, Washington, DC, Federal Reserve Board.

PRADELS, J.L. (1981), L'informatisation des entreprises : qualité, productivité, rentabilité des projets, Paris, Eyrolles.

REIX, R. (1977), La flexibilité de l'entreprise, Paris, Cujas.

RIGAUD, L. (1982), La mise en place des systèmes d'information, Paris, Éditions d'Organisation.

RIVARD, S. et J. TALBOT (1990), Le développement des systèmes d'information : méthodes et outils, Québec, Presses HEC.

RoACH, S.S. (1989), «America's white collar productivity dilemma », Manufacturing Engineering, no 104.

Revue internationale P.M.E., vol. 17, nº 2, 2004

(C) 2004 - Presses de l'Université du Québec

Édifice Le Delta I, 2875, boul. Laurier, bureau 450, Sainte-Foy, Québec G1V 2M2 • Tél. : (418) 657-4399 - www.puq.ca

Tiré de: Revue internationale P.M.E., vol. 17, $\mathrm{n}^{\circ} 2$, sous la direction de Pierre-André Julien • PME1702N

Tous droits de reproduction, de traduction et d'adaptation réservés 
Rowe, F. (1994), «L'impact de l'informatisation sur la performance de l'entreprise», Revue française de gestion, $\mathrm{n}^{\circ} 97$.

SILEM, A. (dir.) (1994), Encyclopédie de l'économie et de gestion, Paris, Hachette Éducation. SOLOw, R. (1987), «We'd better watch out», New York Times Book Review, 12 juillet, p. 36.

SolOw, R. (1988), « Growth theory and after, Nobel lecture », American Economic Review, vol. 78, no 3, p. 307-317.

Strassmann, P.A. (1990), The Business Value of Computers, New Canaan, CT, Information Economic Press.

Strassmann, P.A. (1997), The Squandered Computer, New Canaan, CT, Information Economic Press.

THUROw, L. (1987), «Economic paradigms and slow American productivity growth», Eastern Economy Journal, vol. 13, p. 333-343.

TOUKAM, I. (1999), Analyse des attentes des dirigeants par rapport à l'informatisation des PME au Cameroun, Mémoire de fin de cycle professionnel à l'ESSEC, Université de Douala, Cameroun. 